\title{
LOCAL BUMP METHOD FOR MEASUREMENT OF TRANSVERSE IMPEDANCE OF NARROW-GAP ID CHAMBERS IN STORAGE RINGS*
}

\author{
L. Emery ${ }^{\dagger}$, G. Decker, and J. Galayda \\ Advanced Photon Source, Argonne National Laboratory, Argonne, IL
}

\begin{abstract}
A local bump method has been proposed to determine the transverse impedance of narrow-gap insertion-device (ID) vacuum chambers. As a stored bunch passes through an impedance section, the bunch receives a small transverse kick, which depends on the bunch total charge, the bunch length, and the transverse position. The kick can be measured as a function of orbit by applying a four-magnet bump and forcing closure by running orbit correction. We measured the ID vacuum chambers with apertures of $5 \mathrm{~mm}$ and $8 \mathrm{~mm}$, and a straight section with regular $42 \mathrm{~mm}$ aperture extrusion. Two types of local bumps are applied: a parallel and an angle bump, each of which emphasizes the impedance of different parts of the straight section.
\end{abstract}

\section{INTRODUCTION}

Single-bunch current in light-source storage rings may be limited by instabilities produced by short-term transverse wakefields, mostly produced by small-verticalaperture vacuum chambers in insertion device (ID) straight sections. Our goal is to measure the effect of the installed ID chambers, especially those of $5 \mathrm{~mm}$ aperture. Though one can measure the combined effect of all chambers by measuring the transverse tune shift with single bunch current, it is difficult to accurately measure the change in this tune shift after one or a few new ID chambers are installed. If several identical IDs are installed over a period of time, then one can estimate the contribution of one ID chamber (see the compilation of historical data in refs. [1] and [2]).

The measurement of impedance distribution around a ring has been demonstrated at LEP [3] using phase-advance measurements from beam position monitor (BPM) turn-byturn histories. Workers at LEP were able to fit an average impedance in the long sections of LEP arc and determine the impedance of the rf sections. The method was tried at APS [2]; however, measuring the impedance of a single ID chamber involves only a few BPMs on either side of the chamber, which, in addition to the smallness of the value sought, makes the measurement very noisy.

We report on a sensitive method for measuring ID chamber impedances that uses a local bump to create an effect on the bunch from the wakefields produced in the bump. While traveling through the local bump, the head of the bunch sees no wake force and will follow the normal closed

\footnotetext{
* Work supported by U.S. Department of Energy, Office of Basic Energy Sciences, under Contract No. W-31-109-ENG-38.

$\dagger$ emery@aps.anl.gov
}

orbit of the bump. However, the tail will see some deflecting force from the vacuum chamber proportional to the trajectory offset at every turn, and will be forced to travel along a different orbit from the head. The BPMs of the ring will detect the average particle closed orbit and will read a different orbit from the zero-bunch current case. Rather than allowing the orbit outside the bump to change due to the wake force, we enforce bump closure using orbit correction with only two correctors of the bump. Because the orbit outside the bump is zero, BPM readbacks and some systematic errors are no longer of relevance. The impedance analysis deals with the resulting closed-bump coefficients, rather than with orbit readbacks.

We will present the implementation of the measurement that emphasized the reduction of systematic errors, which would otherwise make the measurement impossible. The results of several types of ID chambers are presented.

\section{IMPLEMENTATION}

We created symmetric four-corrector bumps across the ID straight sections in parallel and angle modes. The parallel bump samples the impedance uniformly across the straight section. The angle bump, with the trajectory crossing the beam axis in the center, is more sensitive to the impedance at the ends of the straight section. The results of the two measurements are used to determine the effects of the impedance of the geometrical tapers (located at the ends of the chamber) and that of the resistive wall, which is uniform along the section.

Bump closure is enforced using feedback to adjust only the middle two correctors to minimize the rms reading on all of the (heavily averaged) BPMs outside the bump. When orbit correction converges, the corrector setpoints may drift a bit due to sources outside the bump, which would otherwise create an orbit on the order of $5 \mu \mathrm{m}$ around the ring.

The deflecting impedance force is linear in the transverse beam position in the bump and the beam charge. Given the smallness of the effect and possible random errors, we collected bump coefficients for several bunch charge and bump amplitude values and fit slopes for the dependencies. We scanned the bump amplitudes with beam stored, waiting each time for orbit correction to converge. The setpoints of the outer two correctors were the independent variables in the scan (rather than the BPM readbacks inside the bump), making comparison with other bunch charge experiments convenient. The same setpoint values for the outer corrector values are used for all bunch patterns. 
Figure 1 shows a simulation of a closed parallel-bump scan from $-1 \mathrm{~mm}$ to $1 \mathrm{~mm}$ in one straight section of the APS. Symbols indicate where the BPM are located, though their readbacks are not used. The inner correctors are just inboard of the middle quadrupole of the triplets. A fake 5-m-long quadrupole was placed in the straight section to take the role of a defocusing impedance. The ratio of the middle correctors to the outer correctors was expected to increase for a defocusing wake by a small amount. The change of the ratio shown here is about $10 \%$, to make the effect visible. The effect observed in the storage ring for the smallest chamber was actually about 0.5\%, 20 times less. If the bump were not forced to be closed in this measurement, the orbit distortion would be about $5 \mu \mathrm{m}$, about the same as the natural orbit drift. These observations show the importance of taking impedance data in such a way to simplify the analysis, and to eliminate random trends.

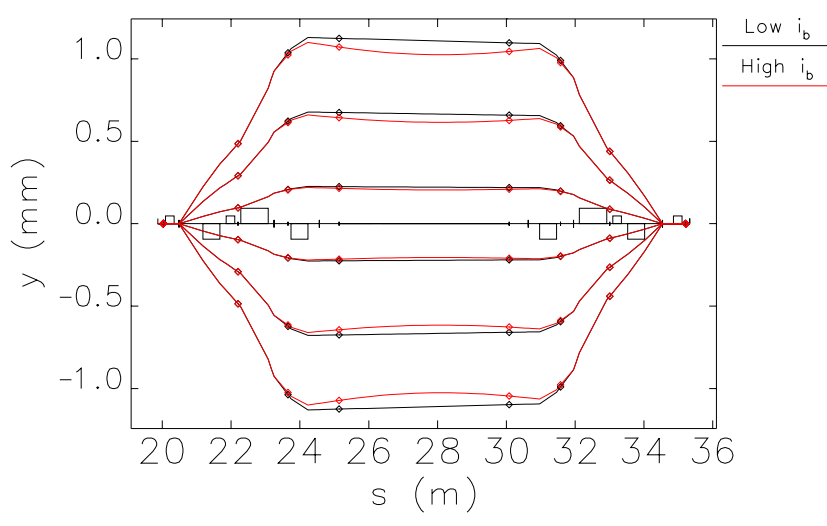

Figure 1: Local bump simulation in a straight section with 20 times impedance signal observed.

We varied the bunch charge while maintaining a total current of $10 \mathrm{~mA}$ distributed around the ring. This avoided changes in thermal effects from synchrotron radiation heating. Because the BPM electronics read the signals only from the bunch under study, there is the possibility of BPM offset changes due to intensity effects. However, the effect is reduced by correcting the orbit after the fill to a standard set of BPM setpoints, transferring the error readback to the setpoints, then starting the closed-bump scan.

There was no need to turn off the sextupoles located in the bump because the orbit through the sextupoles reproduced almost exactly for each bump amplitude scan.

The full range of the correctors is $1.2 \mathrm{mrad}$. Creating a bump of $\pm 1 \mathrm{~mm}$ required about half the full range. There does not seem to be any hysteresis effects, as the bump coefficients under the same beam conditions reproduced well. There were problems with the corrector power supply linearity throughout the range. Fortunately, this systematic error was found to be reproducible in each corrector in each scan, and does not affect the analysis.

\section{MEASUREMENTS}

We denote the setpoint values for the closed-bump correctors in order along the straight section as $I_{1}, I_{2}, I_{3}$, and $I_{4}$, and the bunch charge as $q$. During an experiment, $I_{1}$ and $I_{4}$ (outer correctors) are scanned with the set of values $I_{1, i}$ and $I_{4, i}$, where $i$ is an index internal to the scan. $I_{2, i}^{q}$ and $I_{3, i}^{q}$ are measured and are expected to depend on the bunch charge, $q$. Because of the slight nonlinearity of the corrector output, we did not fit slopes to the $\left(I_{1, i}, I_{2, i}^{q}\right)$ or $\left(I_{1, i}, I_{3, i}^{q}\right)$ points, but rather to the relative changes to $\left(I_{1, i}, I_{3, i}^{q}\right)$ as $q$ is scanned. We took the lowest-charge experiment (say, for $q=q_{0}$ ) as baseline data for all corrector scans, and defined $\Delta I_{2, i}^{q}=I_{2, i}^{q}-I_{2, i}^{q_{0}}$, where it is understood that $\Delta I_{2, i}^{q}=0$ for $q=q_{0}$. Thus, we fit a slope to $\left(I_{1, i}, \Delta I_{2, i}^{q}\right)$, which has smaller fit residuals than the fit of $\left(I_{1, i}, I_{2, i}^{q}\right)$; this demonstrates that the corrector nonlinearities are reproducible. The sum $\Delta I_{2, i}^{q}+\Delta I_{3, i}^{q}$ of the two inner correctors (the difference $\Delta I_{2, i}^{q}-\Delta I_{3, i}^{q}$ is used for an angle bump) is a useful slope since the two correctors together compensate for the kicks produced by the impedance elements in the straight section.

Figure 2 shows the $\Delta I_{2, i}^{q}+\Delta I_{3, i}^{q}$ data for a 5-mm aperture vacuum chamber and four bunch charges. The fitted slopes vary linearly with charge.

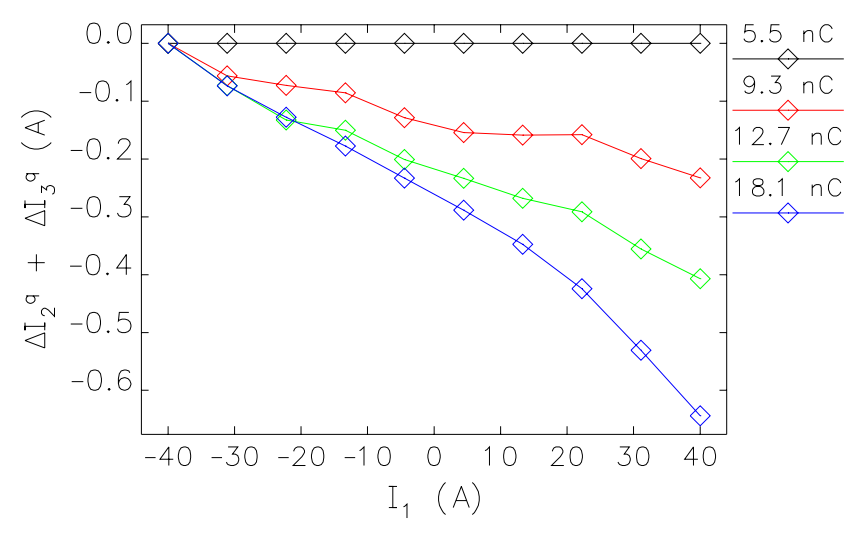

Figure 2: Changes in corrector effort to close a bump for various charges stored in a bunch for a $5-\mathrm{mm}$ aperture vacuum chamber.

We made measurements on two aluminum ID vacuum chambers with $15 \mathrm{~mm} \times 5 \mathrm{~mm}$ elliptical aperture, one ID with $20 \mathrm{~mm} \times 8 \mathrm{~mm}$ elliptical aperture (the standard chamber), and an unused straight section with a large elliptical aperture extrusion of $85 \mathrm{~mm} \times 40 \mathrm{~mm}$. Most measurements were made with only two bunch charges of $6 \mathrm{nC}$ and $18 \mathrm{nC}$. One of the 5-mm-aperture chambers were measured with parallel bumps and angle bumps in an attempt to differentiate the impedances of the taper and the resistive wall. The taper is a copper block that has been machined to reduce the $82 \mathrm{~mm} \times 42 \mathrm{~mm}$ elliptical aperture to match the aperture of the attached ID chamber over an 18-cm length. 


\section{ANALYSIS}

We start with an expression found in [4] (eqn 3.53) for the vertical kick to a trajectory for a bunch transported through an impedance element,

$$
<\Delta y^{\prime}>=-\frac{N r_{o} y_{0}}{\pi \gamma} \int_{0}^{\infty} d \omega \operatorname{Im} Z_{1}^{\perp}(\omega)|\tilde{\rho}(\omega)|^{2},
$$

where $N$ is the number of particles in a bunch, $r_{0}$ is the electron radius, $y_{0}$ is the vertical offset tranjectory, $\gamma$ is the relativistic factor, $Z_{1}^{\perp}$ is the transverse impedance for the area, and $\tilde{\rho}(\omega)$ is the fourier transfrom of the charge density. Because the kicks are small, $y$ is constant through each impedance element for a parallel bump. For an angle bump, the average $y$ is zero, and the overall kick to the trajectory is zero. However, each part of the straight section will contribute a kick proportional to $y$ and produce an offset in the trajectory, which is corrected in the closed bump by an asymmetric adjustment of the inner correctors. The factor of $y_{0}$ in equation 1 acts as an inverse focal length, which suggests the following equation of motion for the straight section with the contribution of the two impedances replaced by focusing terms:

$$
\begin{aligned}
y^{\prime \prime}(s)= & (K l)_{\mathrm{T}}(\delta(s+L / 2)+\delta(s-L / 2)) y(s)+ \\
& \frac{1}{L}(K l)_{\mathrm{RW}} y(s),
\end{aligned}
$$

where $s$ is the longitudinal coordinate with $\mathrm{s}=0$ at the center, $y^{\prime \prime}$ is the second derivative of the trajectory averaged over particles in the bunch, and the $K l$ 's are the integrated focusing strength of the geometrical taper and the resistive wall of the 5-m-long chamber. The $(K l)$ are related to the effective impedance by

$$
(K l)=q \frac{i\left(Z_{1}^{\perp}\right)_{\mathrm{eff}}}{2 \sqrt{\pi} \sigma_{t}(E / e)},
$$

where $\sigma_{t}$ is the bunch time length (we use a constant $\sigma_{t}$ $=30 \mathrm{ps}$ for simplicity) and $E=7 \mathrm{GeV}$. This relation was derived using equations (6.143) and (6.205) in ref. [4].

There exists a linear relation between the $(K l)$ and the corrector strength changes measured in the closed-bump experiments:

$$
\begin{aligned}
& \left(\frac{\Delta I_{2}^{q}+\Delta I_{3}^{q}}{I_{1}}\right)_{\|}=A(K l)_{\mathrm{RW}}+B(K l)_{\mathrm{T}} \\
& \left(\frac{\Delta I_{2}^{q}-\Delta I_{3}^{q}}{I_{1}}\right)_{L}=C(K l)_{\mathrm{RW}}+D(K l)_{\mathrm{T}},
\end{aligned}
$$

where the $A, B, C$, and $D$ coefficients are determined from tracking simulations with elegant [5] reproducing the local bump experiment with orbit correction. It is expected that $2 A \approx B$, since there are two tapers and the trajectory through all impedance elements are the same, and that $C \ll D$, since there is a small average absolute trajectory through the section. Tracking simulations gave the results $A=-4.1 \mathrm{~m}, B=-8.2 \mathrm{~m}, C=-0.4 \mathrm{~m}$, and $D=-2.2 \mathrm{~m}$. The same equation structure applies for the slopes of the relative current quantities with respect to $q$ and for the normalized quantity $(K l) / q$.

For vacuum chambers where no angle bump measurements were made, we use only the first part of equation 4 and the $A$ term to get the total $(K L) / q$. Table 4 shows the results of measurements. Errors are estimated for measurements made more than once. The sensitivity of the measurement of $i\left(Z_{1}^{\perp}\right)_{\mathrm{eff}}$ is about $20 \mathrm{k} \Omega / \mathrm{m}$.

Table 1: Impedance Fitting for Various Aperture Chambers

\begin{tabular}{|c|c|c|}
\hline Element & $\begin{array}{c}d\left(\left(\Delta I_{2}^{q}+\Delta I_{3}^{q}\right) / I_{1}\right)_{\|} / d q \\
\left(\mathrm{~m}^{-1} \mathrm{C}^{-1}\right)\end{array}$ & $\begin{array}{c}i\left(Z_{1}^{\perp}\right)_{\text {eff }} \\
(\mathrm{k} \Omega / \mathrm{m})\end{array}$ \\
\hline $5 \mathrm{~mm}$ (ID3) & $5.4 \pm 0.4 \times 10^{5}$ & $96 \pm 8$ \\
\hline $5 \mathrm{~mm}$ (ID4) & $4.36 \pm 0.8 \times 10^{5}$ & $78 \pm 14$ \\
\hline $8 \mathrm{~mm}$ & $9.4 \times 10^{4}$ & 16 \\
\hline $40 \mathrm{~mm}$ & $1.1 \times 10^{5}$ & 20 \\
\hline
\end{tabular}

Both parallel-bump and angle-bump experiments were performed on one 5-mm chamber (ID4); the results are summarized in Table 4. The measurements were $d\left(\left(\Delta I_{2}^{q}+\Delta I_{3}^{q}\right) / I_{1}\right)_{\|} / d q=5.9 \pm 0.2 \times 10^{5} \mathrm{C}^{-1}$ and $d\left(\left(\Delta I_{2}^{q}-\Delta I_{3}^{q}\right) / I_{1}\right)_{\|} / d q=6 \pm 3 \times 10^{4} \mathrm{C}^{-1}$. For comparison, a calculation of $i\left(Z_{1}^{\perp}\right)$ eff for RW using eqn. 3.57 of reference [4] gives $30 \mathrm{k} \Omega / \mathrm{m}$, a factor 2.5 less than the measurement. Unfortunately, the angle-bump data are highly susceptible to interference by orbit drift, which is responsible for the relatively large uncertainty of the individual contributions but not that of the total focusing strength term.

Table 2: Impedance Component Fitting for a 5-mm-Aperture Chamber

\begin{tabular}{|c|c|c|}
\hline Element & $\begin{array}{c}(\mathrm{Kl}) / q \\
\left(\mathrm{~m}^{-1} \mathrm{C}^{-1}\right)\end{array}$ & $\begin{array}{c}i\left(Z_{1}^{\perp}\right) \text { eff } \\
(\mathrm{k} \Omega / \mathrm{m})\end{array}$ \\
\hline RW & $1.1 \pm 0.2 \times 10^{5}$ & $82 \pm 14$ \\
\hline Taper & $1.4 \pm 1.1 \times 10^{4}$ & $10 \pm 6$ \\
\hline Total & $1.44 \pm 0.04 \times 10^{5}$ & $106 \pm 4$ \\
\hline
\end{tabular}

\section{REFERENCES}

[1] K. Harkay and N. Sereno, "A Potpourri of Impedance Measurment at the Advanced Photon Source Storage Ring," Proceedings of the 1997 Particle Accelerator Conference, Vancouver, Canada, p. 1700 (1998).

[2] K. Harkay et al, "Impedance and the Single Bunch Limit in the APS Storage Ring," Proceedings of the 1999 Particle Accelerator Conference, New York, p. 1644 (1999)

[3] D. Brandt et al, "Measurements of Impedance Distributions and Instability Threshold in LEP," Proceedings of the 1995 Particle Acceleartor Conference, Dallas, p. 570 (1995).

[4] A.W. Chao, Physics of Collective Beam Instabilities in High Energy Accelerators, John Wiley \& Sons (1993).

[5] M. Borland, APS LS-287 (September 2001). http://www.aps.anl.gov/techpub/lsnotes/lsnotesTOC.html 